\title{
A NOVEL RACETRACK MICROTRON ACCELERATING STRUCTURE*
}

\author{
D.V. Kostin ${ }^{\# *}$, V.I. Shvedunov ${ }^{\star}$ N.P. Sobenin ${ }^{\#}$, and W.P. Trower \\ World Physics Technologies, Blacksburg VA
}

\section{Abstract}

We have designed, constructed, and evaluated a $\underline{\text { Rectangular }} \underline{\text { Cavities }} \underline{\text { Biperiodic }} \underline{\text { Structure prototype for }}$ our compact $70 \mathrm{MeV}$ pulsed Race Track $\underline{\text { Microtron [1]. }}$ We present here the design details and test measurements.

\section{INTRODUCTION}

Our mobile $70 \mathrm{MeV}$ RTM requires a narrow accelerating structure to allow $1^{\text {st }}$ orbit electrons to bypass the structure. We solved this problem by using a rectangular accelerating structure with rectangular vertically extended beam slots. This structure also produces high frequency horizontal focusing, which we use to simplify the RTM construction and operation [1,2].

\section{STRUCTURE CALCULATION}

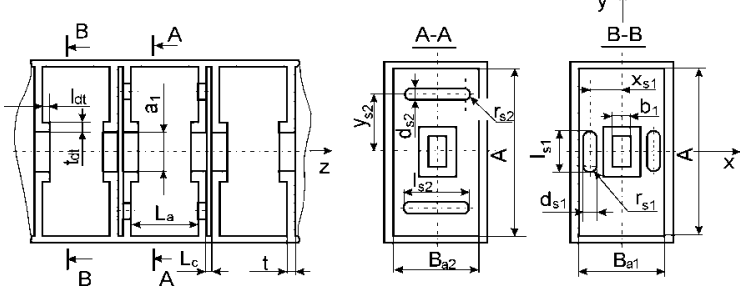

Figure 1: Rectangular cavities biperiodic structure

We found the RCBS structure dimensions with optimal electrodynamics characteristics, seen in Fig. 1, using analytical and numerical calculations as well as computer simulations [3]. We calculated a seven cell section, seen in Fig. 2, whose first cell is for $\beta=0.67$. In Table 1 we present the structure dimensions where the first and last end cells are listed separately. In Fig. 3 we show the calculated RCBS dispersion characteristics. Figure 4 contains the calculated on-axis longitudinal electrical field.

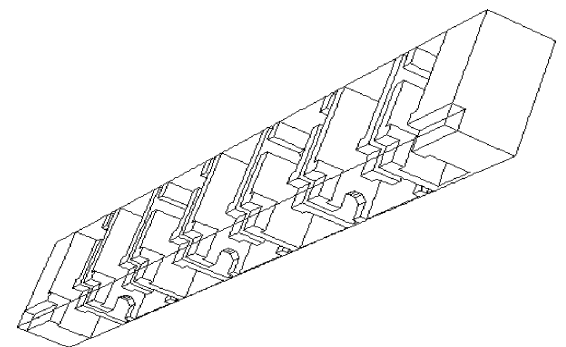

Figure 2: Seven cell RCBS section

"Work supported in part by NSF DMI-9704039

"Permanent address: Electrical Engineering Department, Moscow

Engineering Physics Institute, Moscow, Russia

*Email: wptinc@naxs.net

^Permanent address: Institute of Nuclear Physics, Moscow State University, Moscow, Russia
Table 1: RCBS dimensions (in $\mathrm{mm}$ )

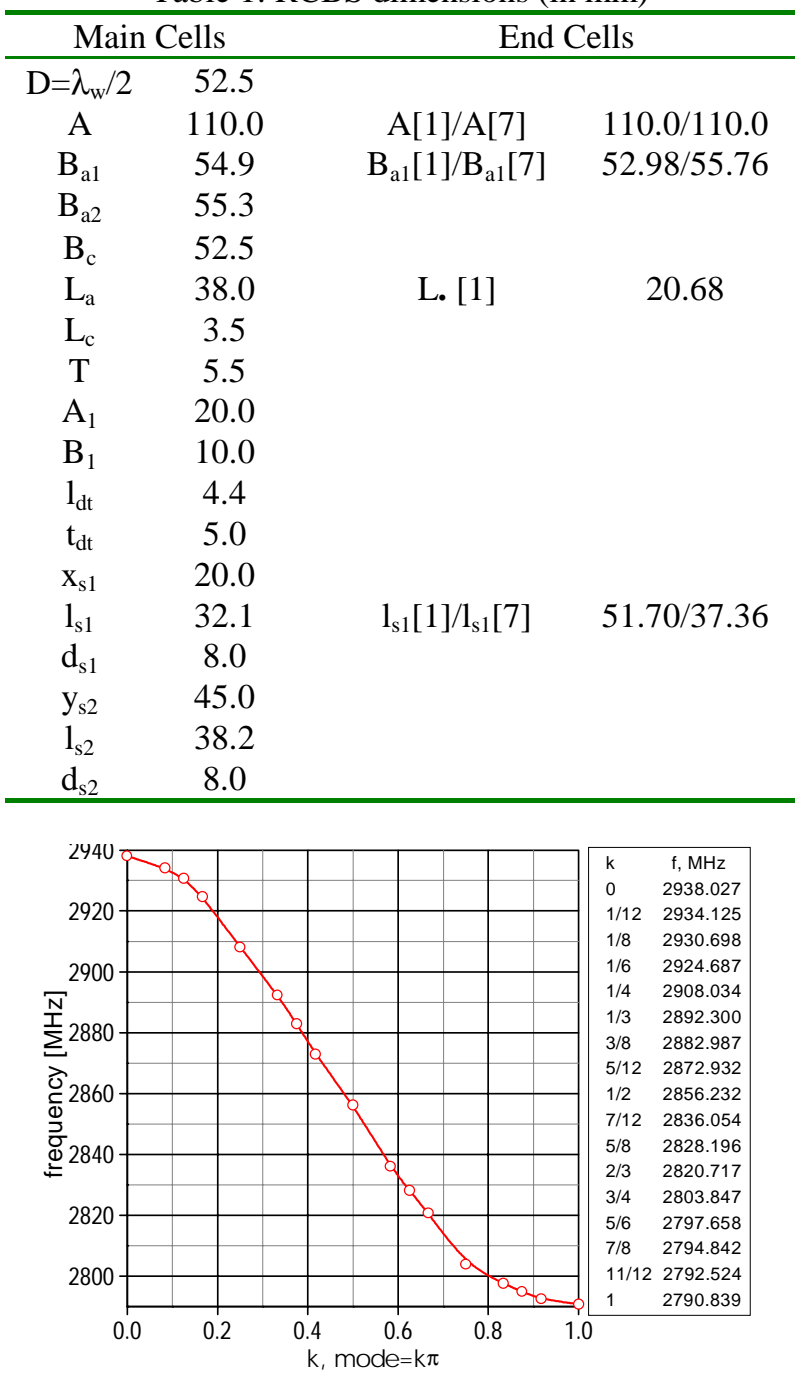

Figure 3: Seven cell RCBS section dispersion characteristics

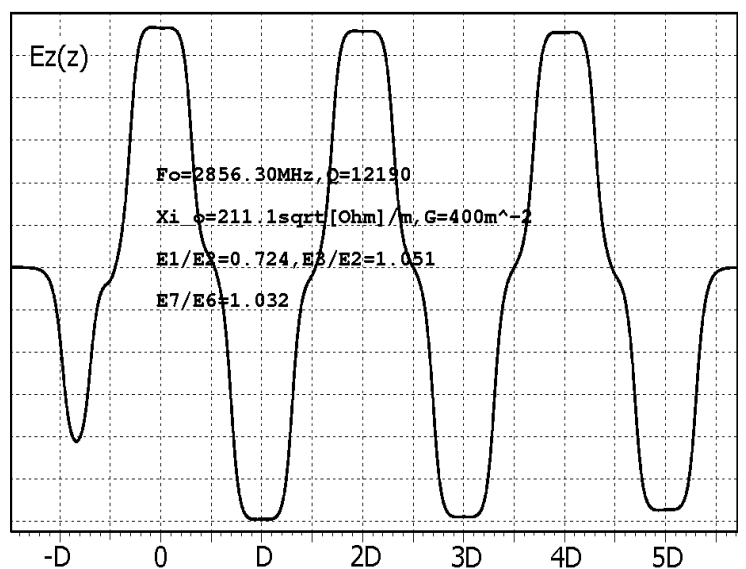

Figure 4: Seven cell RCBS section $\mathrm{E}_{\mathrm{z}}(0,0, \mathrm{z})$ 


\section{STRUCTURE TUNING}

Our manufactured seven accelerating and six coupling cell section geometry was adjusted to obtain the operating frequency in various resonant setups, each with a different number of RCBS periods. We then tuned the end cells to obtain the required accelerating field distribution at the operating frequency by adjusting the end cell coupling slot lengths and narrow wall dimensions, $\mathrm{B}_{\mathrm{a} 1}[\mathrm{i}], \mathrm{i}=1$ and 7 . Next we tuned the coupler cell frequency by decreasing its narrow wall dimension by $0.6 \mathrm{~mm}\left(\mathrm{~B}_{\mathrm{A} . \mathrm{CPL}}=54.3 \mathrm{~mm}\right)$. We chose the coupling window width for the $72 \times 34 \mathrm{~mm}^{2}$ feeding waveguide using its analytical dependence on the normalized impedance, seen in Fig. 5 [4]. To support a $0.7 \mathrm{~A}$ total beam current from all orbits, we matched the coupler using a coupling coefficient of $\sim 3.5$ [4]. An approximate analytical formula gave a window width of $31.7 \mathrm{~mm}$, which we made $28 \mathrm{~mm}$ and increased to $28.8 \mathrm{~mm}$ during tuning.

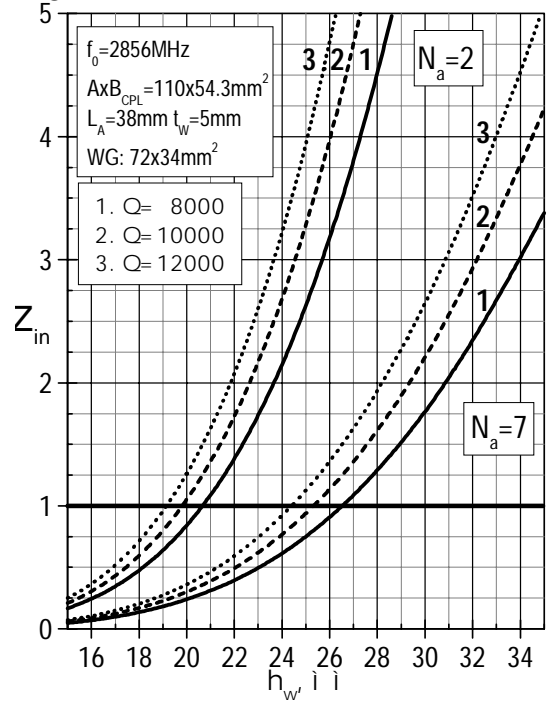

Figure 5: Coupler impedance with window width

\section{STRUCTURE MEASUREMENTS}

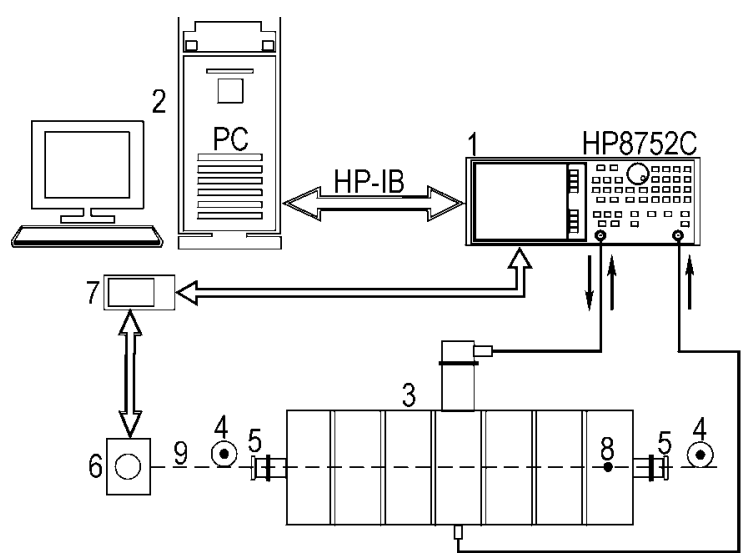

Figure 6: Measurement setup

We measured the section electrodynamics characteristics using the setup shown in Fig. 6, which consisted of (1) a network analyzer, (2) a personal computer, and a mechanical assembly. This assembly included (3) the structure, (4) a bead pulling system with (5) pulleys, (6) thread directors inserted into drift tubes, and (7) a stepper motor/controller. The stepper motor, used to drive the (8) ceramic bead attached to (9) a nylon thread, was controlled by the computer through the network analyzer.

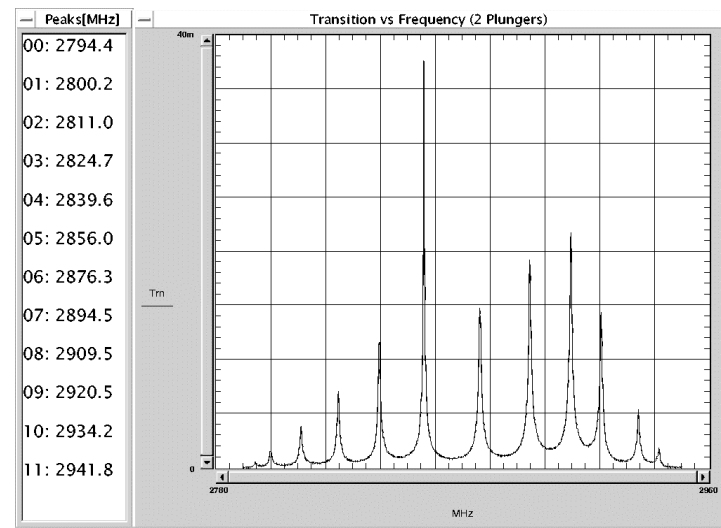

Figure 7: RF modes spectra measurements

After tuning and matching the coupler with the structure, we obtained the $\pi / 2$ mode operating frequency of $f_{0}=(2856 \pm 0.1) \mathrm{MHz}$ at $20^{\circ} \mathrm{C}$ in agreement with the calculated value. We realized the lowest passband $E_{11}$ resonance frequencies, seen in Fig. 7, using both reflection (standing wave ratio) and transition $\left(\mathrm{K}_{\mathrm{tr}}\right)$ coefficient measurements, with two different field exciting and pickoff points, through a waveguide adapter connected to the coupler and through a metal plunger inserted into the drift tube.

After matching the coupler with the rectangular waveguide, the structure coupling coefficient was 3.0. Using a small $5 \mathrm{~mm}$ diameter metal rod mounted near the narrow rectangular waveguide wall, we obtained the 1.0 standing wave ratio used in field measurements. We also measured the Q-factor.

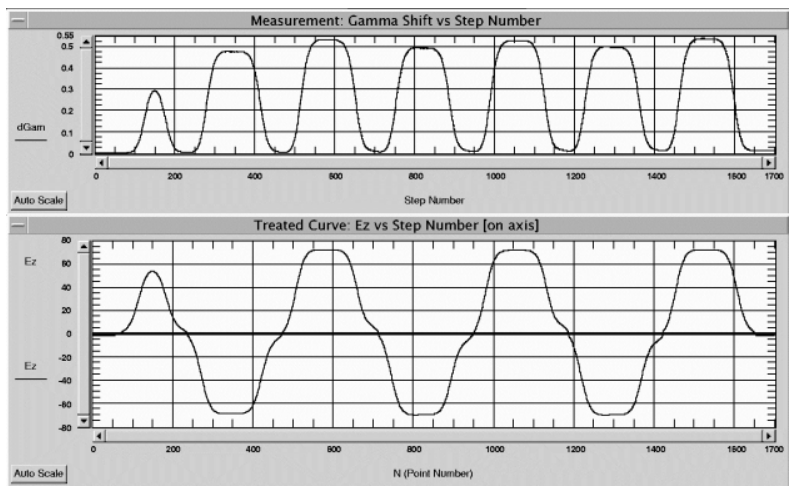

Figure 8: Seven cell $\mathrm{E}_{\mathrm{z}}(0,0, \mathrm{z})$ measured distribution

We measured the field distribution and effective shunt impedance using the bead-pull method with a $6 \mathrm{~mm}$ long, $0.6 \mathrm{~mm}$ diameter dielectric cylinder as the perturbing bead. The bead form factor, measured in the $\mathrm{E}_{010}$ cylindrical 
resonator mode, was $8.849 \times 10^{-20} \mathrm{~m}^{2} \mathrm{c} / \Omega \mathrm{m}$. We made these field distribution measurements, seen in Fig. 8, in two ways. In the first we obtained the shunt impedance by measuring, with the network analyzer, the induced frequency shift as the bead moved along the structure axis. From $\xi(z)=E_{z}(z) / \sqrt{P Q}=\sqrt{\Delta f /\left(2 \pi K_{f}^{E} f_{0}^{2}\right)}$, where $E_{z}(\mathrm{z})$ is the on-axis longitudinal electric field, $P$ is RF power, and $Q$ is the Q-factor, $\xi_{0}=E_{z 0} / \sqrt{P Q}$ was obtained by integrating over the regular part of the field distribution curve and its first harmonic amplitude for $\xi(z)$.

In the second more accurate method, we measured the reflection coefficient, $\Gamma$, at a fixed frequency to obtain the longitudinal electrical field gradient. Here the electric field at the bead position is proportional to $\sqrt{\Delta \Gamma}$, and we calculated the focusing gradient, $G_{X}=2\left(I(x) / I_{0}-1\right) / x^{2}$ (or y-axis). $I_{0}$ is the integral of $\sqrt{\Delta \Gamma(z)}$ over the regular part of the on-axis field data and $I(x)$ is this measurement off-axis by a distance $x$. The measured adjacent cell electrical field amplitude ratios were found to be $\mathrm{E}_{1} / \mathrm{E}_{2}=0.78, \mathrm{E}_{3} / \mathrm{E}_{2}=1.06$, and $\mathrm{E}_{7} / \mathrm{E}_{6}=1.04$.

The effective shunt impedance to Q-factor ratio was obtained from $r_{\text {sheff }} / Q=\xi_{0}^{2} L / 2$, where $L$ is the regular structure length. The test section is not a regular structure so we used an indirect effective shunt impedance measurement. We found $\xi_{0}$ and related it to the effective shunt impedance by $r_{\text {sheff }} / Q \sim \xi_{0}^{2}$. We then obtained the full section shunt impedance to $\mathrm{Q}$ factor, $R / Q$, by integrating $\xi(z)$ over the full section length. The structure electrodynamics characteristics results are seen in Table 2.

Table 2: Structure Electrodynamics Characteristics

\begin{tabular}{lcc}
\hline Characteristic & Measured & Calculated \\
\hline $\mathrm{f}_{0}(\mathrm{MHz})$ & $2,856 \pm 0.1$ & $2,856.0$ \\
$\mathrm{k}_{\mathrm{c}}(\%)$ & 5.1 & 5.1 \\
$\mathrm{Q}$ & $10,800 \pm 200$ & 12,400 \\
$\xi_{0}(\sqrt{\Omega} / \mathrm{m})$ & $205 \pm 5$ & 211 \\
$\mathrm{r}_{\text {sh,eff }} / \mathrm{Q}(\mathrm{k} \Omega / \mathrm{m})$ & $3.3 \pm 0.3$ & 3.65 \\
$\mathrm{r}_{\text {sh,eff }}(\mathrm{M} \Omega / \mathrm{m})$ & $37 \pm 3$ & 45 \\
$\mathrm{R} / \mathrm{Q}(\mathrm{k} \Omega)$ & $1.25 \pm 0.02$ & 1.23 \\
$\mathrm{G}_{\mathrm{x}}\left(\mathrm{m}^{-2}\right)$ & $500 \pm 100$ & 410 \\
$\mathrm{G}_{\mathrm{y}}\left(\mathrm{m}^{-2}\right)$ & $-300 \pm 100$ & -410 \\
\hline
\end{tabular}

\section{CONCLUSIONS}

The RCBS for our compact $70 \mathrm{MeV}$ pulsed RTM has measured EDC in good agreement with those we calculated. Our RCBS, which operates in the $\pi / 2$ mode at $2,856 \mathrm{MHz}$ with a $\mathrm{Q}$ of 11,000 , has a $\sim 40 \mathrm{M} \Omega / \mathrm{m}$ effective shunt impedance and a $\sim 400 \mathrm{~m}^{-2}$ focusing gradient.

\section{REFERENCES}

[1] E.A. Knapp, A.W. Saunders, V.I. Shvedunov, N.P. Sobenin and W.P. Trower, "A Mobile Racetrack Microtron", Nucl. Instrum. Meth. B139 (1998) 517.

[2] D.V. Kostin, V.N. Melekhin, V.I. Shvedunov, N.P. Sobenin, and W.P. Trower, "High Frequency Focusing-Accelerating Structures", in Applications of Accelerators in Research and Industry, J.L. Dugan, and I.L. Morgan, eds. (AIP Press, Woodbury, 1997) vol. CP392, p.1135.

[3] R. Klatt, F. Krawczyk, W.R. Novender, C. Palm, and T. Weiland, "A Three Dimensional Electromagnetic CAD System for Magnets, RF Structures and Transient Wake-Field Calculations", in Proc. 1986 Linear Accelerator Conf., SLAC-303 (1986) p.276.

[4] N.P. Sobenin and B.V. Zverev, Electrodynamic Characteristics of Accelerating Cavities, (Energoatomizdat, Moscow, 1993) 220pp. (in Russian). 